\title{
Markovian Approach for the Analysis and Prediction of Weekly Rainfall Pattern in Makurdi, Benue State, Nigeria
}

\section{*11LAWAL, ADAMU; ABUBAKAR, UY; DANLADI, HAKIMI; ANDREW, SABA GANA}

\author{
${ }^{I}$ Department of Mathematics, Federal University of Technology Minna, Nigeria \\ ${ }^{2}$ Department of Mathematics, Federal University of Technology Minna, Nigeria \\ ${ }^{3}$ Department of Mathematics, Federal University of Technology Minna, Nigeria \\ ${ }^{4}$ Department of Crop Production, Federal University of Technology Minna, Nigeria \\ E-mail:lawal.adamu@futminna.edu.ng)E-mail:abbkruy@yahoo.com; E-mail:hakimi_shengu@yahoo.com \\ E-mail:andrewganasaba@yahoo.com
}

\begin{abstract}
A stochastic model to study weekly rainfall pattern has been presented in this paper. The Markovian method was used to predict and analyze weekly rainfall pattern of Makurdi, Benue state, Nigeria for a period of eleven years (2005-2015). After some successful iterations of the model, its stabilizes to equilibrium probabilities, revealing that in the long-run $22 \%$ of the weeks during rainy season in Makurdi, will experience no rainfall , $50 \%$ will experience low rainfall, $25 \%$ will experience moderate rainfall and $2 \%$ will experience high rainfall. The model also reveals that, a week of high rainfall cannot be followed by another week of high rainfall, a week of high rainfall cannot be followed by a week of no rainfall, and a week of moderate rainfall cannot precede a week of high rainfall. These results are important information to the residents of Markudi and environmental management scientists to plan for the uncertainty of rainfall. @ JASEM
\end{abstract}

\section{http://dx.doi.org/10.4314/jasem.v20i4.8}

Keywords: Markov chain, Weekly Rainfall, Transition Probabilities, Equilibrium probabilities Probability State Vector, Makurdi.

Rainfall exhibits a strong variability in time and space across the globe. It is well established that rainfall is changing on both the global and the regional scale due to global warming (Hulmeet al., 1998). Rainfall is the principal phenomenon driving many hydrological extremes such as floods, droughts, landslides, debris and mud-flows; its analysis and modeling are typical problems in applied hydrometeorology (Barkotulla, 2010). Hence, its stochastic modeling is necessary for the prevention of natural disaster. Understanding the rainfall distribution is equally necessary for future planning. This is applicable in areas like agriculture, industry, insurance, hydrological studies and the entire planning of a country economy. Consequently, information on rainfall probabilities is vital for the design of water supply management, supplementary irrigation schemes and the evaluation of alternative cropping system for effective soil water management plans (Barkotulla,2010). Such information can also be beneficial in determining the best adapted plant species and the optimum time of seedling to reestablish vegetation on deteriorated rangelands. The aim of this research is to analyse and predict weekly rainfall pattern of Makurdi in the following week, subsequent weeks and in the long run, given the rainfall in the present week. The results from the model could be useful to the residents of Makurdi for effective planning and viable production.
Overwhelming researchers within Nigeria and in around the world, have proposed several methods in attempt to provide information that could enable humanity to make best use of this random phenomenon, either for agricultural purposes or other purposes of fundamental importance to life, such researchers include. Raheem et al., (2015) had successfully developed a three-state Markov chain to examine the pattern and distribution of daily rainfall in Uyo metropolis of Nigeria using 15 years (19952009) rainfall data. Evaluation of the effect of climate change on daily rainfall using first-order Markov chain model, have been presented by (Chulsanget al., 2016), the model was applied to Seoul weather station in Korea, the result shows that about $30 \%$ of the total change in monthly rainfall amount was due to the change in the number of wet days and the remaining $70 \%$ was due to the change in the rainfall intensity. Arumugam and $\operatorname{Karthik(2016)}$ have investigated the variations of annual rainfall in Tirunelveli district, India based on stochastic method. Rainfall data for 44 years was used, the Markov chain model developed was used to predict annual rainfall for the future years, up to 2025. Katrin et al., (2016) had applied Markov chains to analyzed the dynamics and succession of multivariate or compound extreme events. The method was applied to observational data and an ensemble of regional climate simulations for Central Europe. They 
concluded that, the change in the succession of hot and dry days in summer will probably affect regions in Spain and Bulgaria. The susceptibility to a dynamic change of hot and dry extremes in the Russian region will probably decrease .Mouelhi et al.(2016) had proposed a developmental method of stochastic generator of monthly rainfall series. The work was based on the modeling of the occurrence and the quantity of rain in a separate way.

\section{MATERIALS AND METHODS}

Study Area :The study area of this research is Makurdi. The city is located in central Nigeria along the Benue River, it is located on (latitude $7.7^{0} \mathrm{~N}$, longitude $8.5^{\circ}$ ), Makurdi is the capital city of Benue state of Nigeria. Benue State is regarded as food basket of the nation because of its rich agricultural produce which include Yam, Rice, Beans, Cassava, Sweet-potato, Maize, Soybean, Sorghum, Millet, Sesame, cocoyam etc. Agriculture is the mainstay of the economy, engaging over $75 \%$ of the state farming population. The State also has one of the longest stretches of river systems in the country with great potential for a viable fishing industry, dry season farming through irrigation and for an inland water highway. The vegetation of the State consists of rain forests which have tall trees, tall grasses and oil palm trees that occupy the state's western and southern fringes while the Guinea Savannah is found in the eastern and northern parts with mixed grasses and trees that are generally of average height.

Sample Collection: The data used in this research work were obtained from the archive of Nigerian Meteorological Agency, Maitama, Abuja. It is the daily rainfall record of Makurdi, Benue state for the period of 11 years (2005 to 2015).

Model Formulation: This research, considers the use of weekly rainfall amount during the rainy season to study weekly rainfall pattern of Makurdi. In this model, we attempt to analyse and predict weekly rainfall pattern in the long run.

Following Ross (1989), we consider a stochastic process $\left\{X_{n}, \quad n=0,1,2,3, \ldots \ldots ..\right\}$ which takes on a finite or countable number of possible values. Unless otherwise stated, this set of possible values of the process will be denoted by the set of nonnegative integers $\{0,1,2,3, \ldots \ldots \ldots\}$ if $X_{n}=i$ then the process is said to be in state $i$ at time $n$.

Data Evaluation: We suppose that whenever the process is in state $i$, there is a fixed probability $P_{i j}$ that it will next be in state $\mathrm{j}$. That is, we suppose that

$$
P\left\{X_{n+1}=j \mid X_{0}=i_{0}, \ldots, X_{n-2}=i_{n-2}, X_{n-1}=i_{n-1}, X_{n}=i\right\}=P_{i j} .
$$

for all states $i_{0}, i_{i}, \ldots \ldots . ., i_{n-1}, i, j$ and all $n \geq 0$. Such a stochastic process is known as a Markov chain.Equation 1,may be interpreted as stating that, for a Markov chain, the conditional distribution of any future state $X_{n+1}$ given the past states $X_{0}, X_{1}, \ldots \ldots ., X_{n-1}$ and the present state $X_{n}$ is independent of the past states and depends only on the present state. The value $\mathrm{P}_{\mathrm{ij}}$ represents the probability that the process will, when in state $i$, next make a transition into state $\mathrm{j}$. Since probabilities are nonnegative and since the process must make a transition into some state, we have that

$$
P_{i j} \geq 0, \quad i, j \geq 0 ; \quad \sum_{j=0}^{\infty} P_{i j}=1, \quad i=0,1,2 \ldots \ldots \ldots
$$

Now, suppose that the amount of weekly rainfall in Makurdi in a week during the rainy season is considered as a random variable $X$, the collection of these random variables over the weeks constitutes a stochastic process

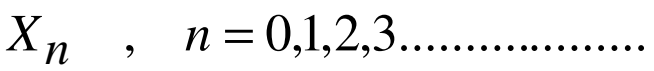

It is assumed that this stochastic process satisfies Markov properties mentioned in the previous section. Let the weekly rainfall be modelled by four states, Markov model.

State1: No rainfall

State2: Low rainfall

State3: Moderate rainfall

State4: High rainfall 
It is important to mention here that, the classification of states for a Markov model is guided by the purpose in which the model is intended to achieve.

The transition between the states is described by Equation 2,thus we have

Transition probability Matrix

$$
p=\left[\begin{array}{cccc}
P_{11} & P_{12} & P_{13} & P_{14} \\
P_{21} & P_{22} & P_{23} & P_{24} \\
P_{31} & P_{32} & P_{33} & 0 \\
0 & P_{42} & P_{43} & 0
\end{array}\right]
$$

Now, Let $P^{(n)}$ represent the probability state vectors of the Markov chain,

Where $n$ can take values from zero to infinity $(\mathrm{n}=0,1,2,3,4, \ldots \ldots \ldots \ldots \ldots \ldots \ldots . .$.

and $P_{i}^{(n)}$ is the probability that the weekly rainfall is in the $i^{\text {th }}$ state at the $n^{\text {th }}$ week. $P^{(0)}$ is the initial state vector of the Markov chain and $P^{(n)}$ is the state vector at the $n^{\text {th }}$ week

Then we can write $p^{(n+1)}=P^{(n)} P(3)$

Where $\mathrm{P}$ is our transition probability matrix and $p^{(n+1)}$ is the state vector at the $(n+1)^{t h}$ week. on iteration, we have

$p^{(n)}=p^{(0)} P^{n}(4)$

this mean that the initial state vector $P^{(0)}$ and the transition matrix P determine the state vector $P^{(n)}$ at the $n^{\text {th }}$ week

If we now, let $p^{(n)}=\left\lfloor\begin{array}{llll}p_{1}^{n} & p_{2}^{n} & p_{3}^{n} & p_{4}^{n}\end{array}\right\rfloor_{(5)}$

denote the probabilities of finding the weekly rainfall amount in any of the four states at the $n^{\text {th }}$ week and also let $p^{(0)}=\left\lfloor\begin{array}{llll}p_{1}^{o} & p_{2}^{o} & p_{3}^{o} & p_{4}^{o}\end{array}\right\rfloor_{(6)}$

Denotes the initial state vector, then our First order Markov Chain Model for weekly rainfall amount pattern prediction in the long run, in Makurdi can be represented by

$$
\left[\begin{array}{llll}
p_{1}^{n} & p_{2}^{n} & p_{3}^{n} & p_{4}^{n}
\end{array}\right]=\left[\begin{array}{llll}
p_{1}^{o} & p_{2}^{o} & p_{3}^{o} & p_{4}^{o}
\end{array}\right]\left[\begin{array}{cccc}
p_{11} & p_{12} & p_{13} & p_{14} \\
p_{21} & p_{22} & 23 & p_{24} \\
p_{31} & p_{32} & p_{33} & 0 \\
0 & p_{42} & p_{43} & 0
\end{array}\right]^{n}
$$

Limiting State Probabilities: The state occupation probabilities is independent of the starting state of the process, if number of the time the state transition is large thus the process reaches a steady state after a sufficiently large period of time. This is equilibrium distribution

$\pi=\left(\begin{array}{llll}\pi_{1} & \pi_{2} & \pi_{3} & \pi_{4}\end{array}\right)$

If we let $n \rightarrow \infty$ in equation 4 , we have

$\pi=\pi$ 
and also $\pi=\sum_{i=1}^{4} \pi_{i}=1$

these equations will be use to find the limiting state probabilities for our model

\section{RESULTS AND DISCUSSION}

Table 1: A summary of weekly rainfall amount in Makurdi

between 2005-2015 and states distribution

\begin{tabular}{lll}
\hline Weekly rainfall in $\mathrm{mm}$ & Frequency & State \\
\hline 0 & 68 & No Rainfall \\
Rainfall amount $<61$ & 254 & Low Rainfall \\
$61-120$ & 51 & Moderate Rainfall \\
Rainfall amount $>120$ & 9 & High Rainfall \\
\hline
\end{tabular}

The transition count matrix is shown below and is obtained from Table 1

$$
M=\left[\begin{array}{cccc}
29 & 25 & 2 & 2 \\
26 & 161 & 47 & 7 \\
4 & 4 & 10 & 0 \\
0 & 6 & 2 & 0
\end{array}\right] \text { (10) }
$$

From equation10, using the maximum likelihood estimator i.e

$$
p_{i j}=\frac{f_{i j}}{\sum_{j=1}^{4} f_{i j}} \quad \text { ij=1,2,3,4 }
$$

Where $f_{i j}$ is the historical frequency of transition from state $i$ to state $j$, we to obtained the transition probability matrix given below

$$
P=\left[\begin{array}{cccc}
0.5 & 0.431 & 0.034 & 0.034 \\
0.108 & 0.668 & 0.195 & 0.029 \\
0.222 & 0.222 & 0.555 & 0 \\
0 & 0.75 & 0.25 & 0
\end{array}\right]_{(12)}
$$

The state of the process at time $\mathrm{n}, \mathrm{Xn}$ is related to the process at time $\mathrm{n}+1$ through what is known as the transition probabilities. If the process is in state $\mathrm{i}$ at time $\mathrm{n}$, at next time step $\mathrm{n}+1$, it will either stay in state $\mathrm{i}$ or move or transfer to another state $\mathrm{j}$.The probabilities for these changes in state are defined by Equation 1, called One-Step transition probabilities.

n-Step Transition Probability: The probability of moving from state $\mathrm{i}$ to state $\mathrm{j}$ in $\mathrm{n}$ times steps is called $\mathrm{n}$-step transition probability and is defined by $p_{i j}^{(n)}=\operatorname{prob}\left\{X_{n}=j / X_{0}=i\right\} \operatorname{Linda}(2010)$

Calculating $P^{n}$, we have, on iteration 


$$
\begin{aligned}
& P^{2}=\left[\begin{array}{llll}
0.304 & 0.536 & 0.128 & 0.029 \\
0.169 & 0.558 & 0.249 & 0.023 \\
0.258 & 0.367 & 0.359 & 0.014 \\
0.137 & 0.556 & 0.285 & 0.022
\end{array}\right]_{(13)} \\
& P^{4}=\left[\begin{array}{llll}
0.221 & 0.526 & 0.227 & 0.024 \\
0.214 & 0.506 & 0.257 & 0.022 \\
0.235 & 0.483 & 0.258 & 0.021 \\
0.212 & 0.500 & 0.265 & 0.021
\end{array}\right]_{(14)} \\
& P^{6}=\left[\begin{array}{llll}
0.218 & 0.508 & 0.248 & 0.022 \\
0.220 & 0.504 & 0.252 & 0.022 \\
0.223 & 0.502 & 0.249 & 0.022 \\
0.221 & 0.502 & 0.253 & 0.022
\end{array}\right]_{(15)} \\
& P^{8}=\left[\begin{array}{llll}
0.220 & 0.504 & 0.250 & 0.022 \\
0.220 & 0.504 & 0.251 & 0.022 \\
0.220 & 0.503 & 0.250 & 0.022 \\
0.221 & 0.503 & 0.251 & 0.022
\end{array}\right]_{(16)} \\
& P^{10}=\left[\begin{array}{llll}
0.219 & 0.503 & 0.249 & 0.022 \\
0.220 & 0.504 & 0.250 & 0.022 \\
0.220 & 0.503 & 0.250 & 0.022 \\
0.220 & 0.504 & 0.250 & 0.022
\end{array}\right]_{(17)} \\
& P^{16}=\left[\begin{array}{llll}
0.219 & 0.502 & 0.249 & 0.022 \\
0.219 & 0.502 & 0.249 & 0.022 \\
0.219 & 0.501 & 0.249 & 0.022 \\
0.219 & 0.502 & 0.249 & 0.022
\end{array}\right] \\
& P^{18}=\left[\begin{array}{llll}
0.219 & 0.501 & 0.249 & 0.022 \\
0.219 & 0.501 & 0.249 & 0.022 \\
0.219 & 0.501 & 0.249 & 0.022 \\
0.219 & 0.502 & 0.249 & 0.022
\end{array}\right] \text { (19) } \\
& P^{18}=\left[\begin{array}{llll}
0.22 & 0.50 & 0.25 & 0.02 \\
0.22 & 0.50 & 0.25 & 0.02 \\
0.22 & 0.50 & 0.25 & 0.02 \\
0.22 & 0.50 & 0.25 & 0.02
\end{array}\right] \text {. corrected to } 2 \text { decimal places }
\end{aligned}
$$

Limiting State Probabilities: The state occupation probabilities is independent of the starting state of the process, if number of the time the state transition is large thus the process reaches a steady state after a sufficiently large period of time. Thus

LAWAL, ADAMU; ABUBAKAR, UY; DANLADI, HAKIMI; ANDREW, SABA GANA 
As $n$ increases, $P^{n}$ gets closer and closer to equation 19, that is $n \geq 18$ the transition probabilities stabilises to equation 19 , and from equation 4 , with the initial state probability vector $\left(\begin{array}{llll}1 & 0 & 0 & 0\end{array}\right)$ we have

$$
\begin{aligned}
& P^{n}=\left(\begin{array}{llll}
1 & 0 & 0 & 0
\end{array}\right)\left[\begin{array}{llll}
0.219 & 0.501 & 0.249 & 0.022 \\
0.219 & 0.501 & 0.249 & 0.022 \\
0.219 & 0.501 & 0.249 & 0.022 \\
0.219 & 0.501 & 0.249 & 0.022
\end{array}\right] .=\left(\begin{array}{llll}
0.219 & 0.501 & 0.249 & 0.022
\end{array}\right) \\
& =\left(\begin{array}{lllll}
0.22 & 0.5 & 0.25 & 0.02
\end{array}\right) \text { corrected } \text { to } 2 \text { decimal places }
\end{aligned}
$$

This is the probability of finding the weekly rainfall amount fall in any of the four states for large $n($ i.e $\quad n \geq 18)$

From equation 8 ,the limiting state probability vector is given by

$$
\pi=\pi P=\left(\begin{array}{llll}
0.22 & 0.50 & 0.25 & 0.02
\end{array}\right)
$$

Where $\pi$ correspond to equation, 19

This shows that in the long-run, $22 \%$ of the weeks during rainy season in Makurdi will experience No rainfall, $50 \%$ will experience Low rainfall, $25 \%$ will experience Moderate rainfall,2\% will experience High rainfall

The research, has applied the principle of Markov in discrete time to study weekly rainfall amount in Makurdi. In the transition probability matrix, we have $\mathrm{P}_{11}, \mathrm{P}_{12}, \mathrm{P}_{13}$, and $\mathrm{P}_{14}=0.50,0.431,0.034$ and 0.034 respectively for the first year, after some iteration; these values stabilised to $0.219,0.501,0.249$ and 0.022 respectively at the 18 steps. These are the equilibrium probabilities. That is, the probabilities of having weekly rainfall in state 1 , state 2 , state 3 and state4 respectively in the lung run. For instance, the value of $\mathrm{P}_{13}$ increases steadily from 0.034 for the first year and stabilised to 0.249 at the 18 steps. This is probabilities of having no rainfall week during the rainy season making a transition to moderate rainfall in the following week or subsequent weeks cannot be more than 0.249. Similar interpretation is given to $\mathrm{P}_{12}$, and $\mathrm{P}_{14}$ as well as to the other transition probabilities. With this Markov chain model, it is easy to make a prediction of what the rainfall (state) may be in the following week and in the long run if we know the present rainfall state on a discrete time scale.

From equation2, it can be observed that $\mathrm{P}_{41}, \mathrm{P}_{34}, \mathrm{P}_{44}$ $=0$, these mean that, there are not transition between these states. For example for the $\mathrm{P}_{44}=0$, it means that, it is not possible for a week of high rainfall in Makurdi to be followed by another week of high rainfall, and also for the $\mathrm{P}_{41}$, it is not possible to for a week of high rainfall to be followed by a week of no rainfall. Similarly, for $\mathrm{P}_{34}$, a week of moderate rainfall cannot precede a week high Rainfall.

Conclusions: A stochastic model to analyze and predict weekly rainfall pattern of Makurdi, Benue state has been presented. The stochastic model was formulated based on the principle of Markov. The results from the model are an important information, that could assists the residents to better understand the dynamics of weekly rainfall in Makurdi which may be helpful for effective planning and viable production.

\section{REFERENCE}

Arumugam, P ;Karthik, SM (2016).Stochastic Modelling in Yearly Rainfall at Tirunelveli District,Tamil Nadu, India. International Conference on Processing of Materials, Minerals and Energy

(July 29th - 30th) 2016, Ongole, Andhra Pradesh, India.

Barkotulla,M(2010). Stochastic Generation of the Occurrence and Amount of Daily Rainfall.

Pakistan Journal of Statistics and Operation Research, 6(2).

Chulsang,Yoo; Jinwook,Lee; Yonghun, Ro(2016).Markov Chain Decomposition of Monthly Rainfall intoDaily Rainfall: Evaluation of Climate Change Impact. Hindawi Publishing CorporationAdvances in Meteorology volume 2016,P.1-10.

Dilek, Eren; Akyuz, Mehmetcik; Bayazit, Bihrat Onoz (2011).Markov Chain Models for 
Hydrological Drought Characteristics.Journal of Hydrometeorology 13: 298-309

Hulme, M; Osborn, T; John, T (1998). Precipitation Sensitivity of Global Warming: Comparison of Observations with HADCM2 Simulations. Geophysical Research Letter, 25, 3379-3382

Katrin, Sedlmeier; Sebastian, Mieruch; Gerd, Schädler; Christoph, Kottmeier (2016).Compound Extremes in a Changing Climate - A Markov Chain Approach.Nonlinear Processes in Geophysics Discussions

Linda, J.S; Allen.(2010). An introduction to Stochastic processes with Application to Biology. Texas, USA: Chapman and Hall.
Mouelhi, Safouane; Nemri, Saida; Jebari, Sihem; Slimani,Mohamed(2016).Using the Markov Chainfor the Generationof Monthly Rainfall Series in aSemi-Arid Zone.Open Journal of Modern Hydrology 6 :51-65

Raheem, M. A; W.B, Yahya; K.O, Obisesan(2015). A Markov Chain Approach on Pattern of Rainfall Distribution.Journal of Environmental Statistics Vol 7 issue 1

Ross S.M. (1989). "Introduction to probability Models" Academic Press, Ltd, London. 